\title{
Caracterización de Pacientes Caninos de Raza Pura Con Diagnóstico de Enfermedad Metabólica Atendidos en el Hospital Veterinario de la Universidad de San Carlos de Guatemala
}

By López-Rivera, Sofía Isabel, Laparra-Galindo, Julia Patricia, Chávez-López, Juan José \& Villatoro-Chacón, Daniela Mariel Resumen- Los perros de raza pura han brindado información sobre la morfología, el comportamiento y las enfermedades caninas siendo la diversificación de las mismas lo que ha llevado al desarrollo de enfermedades metabólicas, las cuales son causadas por anormalidades en sistemas enzimáticos. El presente estudio fue realizado en el Hospital Veterinario de la Facultad de Medicina Veterinaria y Zootecnia de la Universidad de San Carlos de Guatemala en el cual se evaluaron 406 fichas clínicas de pacientes caninos de raza pura de las cuales 48 eran correspondientes con un diagnóstico definitivo de enfermedad metabólica. De los pacientes evaluados, las cuatro razas de mayor presencia fue el Labrador Retriever 17\%, Husky Siberiano13\%, French Poodle 10\% y Schnauzer 8\%.

Palabras clave: canino, raza pura, genética, metabolismo, obesidad, hipotiroidismo, urolitiasis, diabetes mellitus.

GJMR-G Classification: NLMC Code: WA 360

Strictly as per the compliance and regulations of:

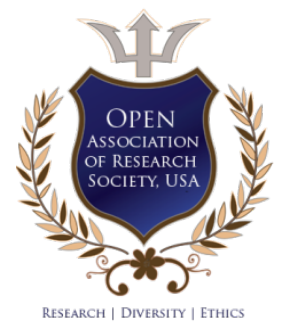

(c) 2019. López-Rivera, Sofía Isabel, Laparra-Galindo, Julia Patricia, Chávez-López, Juan José \& Villatoro-Chacón, Daniela Mariel. This is a research/review paper, distributed under the terms of the Creative Commons Attribution-Noncommercial 3.0 Unported License http://creativecommons.org/licenses/by-nc/3.0/), permitting all non-commercial use, distribution, and reproduction in any medium, provided the original work is properly cited. 


\title{
Caracterización de Pacientes Caninos de Raza Pura Con Diagnóstico de Enfermedad Metabólica Atendidos en el Hospital Veterinario de la Universidad de San Carlos de Guatemala
}

\author{
López-Rivera, Sofía Isabel ${ }^{\alpha}$, Laparra-Galindo, Julia Patricia ${ }^{\circ}$, Chávez-López, Juan José $^{\circ}$ \\ \& Villatoro-Chacón, Daniela Mariel ${ }^{\omega}$
}

\begin{abstract}
Resumen- Los perros de raza pura han brindado información sobre la morfología, el comportamiento y las enfermedades caninas siendo la diversificación de las mismas lo que ha llevado al desarrollo de enfermedades metabólicas, las cuales son causadas por anormalidades en sistemas enzimáticos. El presente estudio fue realizado en el Hospital Veterinario de la Facultad de Medicina Veterinaria y Zootecnia de la Universidad de San Carlos de Guatemala en el cual se evaluaron 406 fichas clínicas de pacientes caninos de raza pura de las cuales 48 eran correspondientes con un diagnóstico definitivo de enfermedad metabólica. De los pacientes evaluados, las cuatro razas de mayor presencia fue el Labrador Retriever 17\%, Husky Siberiano13\%, French Poodle10\% y Schnauzer 8\%. Entre las enfermedades metabólicas observadas en el estudiose encontraron: la obesidad y el hipotiroidismo en un $27 \%$ cada una, enfermedad renal $25 \%$, urolitiasis $17 \%$ y diabetes mellitus $4 \%$.

El propósito del estudio fue obtener una perspectiva general de la predisposición racial de desórdenes metabólicos en caninos de raza pura así como la relación clínica que estos tienen con otros factores tales como la condición corporal, estado reproductivo y sexo.
\end{abstract}

Palabras clave: canino, raza pura, genética, metabolismo, obesidad, hipotiroidismo, urolitiasis, diabetes mellitus.

Abstract- Purebred dogs have provided information on the morphology, behavior and canine diseases being the diversification of them which has led to the development of metabolic diseases, which are caused by abnormalities in enzymatic systems. The present study was carried out in the Veterinary Hospital of the Faculty of Veterinary Medicine and Zootechnics of the University of San Carlos of Guatemala in which 406 clinical records of purebred dogs were evaluated, of which 48 were for a definitive diagnosis of disease metabolic Of the patients evaluated, the four races with the greatest presence were the Labrador Retriever $17 \%$, Siberian Husky $13 \%$, French Poodle 10\% and Schnauzer $8 \%$. Among the metabolic diseases observed in the study were: obesity and hypothyroidism in $27 \%$ each, kidney disease $25 \%$, urolithiasis $17 \%$ and diabetes mellitus $4 \%$.

The purpose of the study was to obtain a general perspective of the racial predisposition of metabolic disorders in dogs of the breed, as well as the clinical relationship with other factors such as body condition, reproductive status and sex.

Author: e-mail: sofisa_riv93@hotmail.com

\section{INTRODUCCIÓN}

os seres humanos han ejercido distintas selecciones en los perros desde su domesticación como lobos hasta la diversidad de razas que conocemos actualmente. A lo largo de esto, ha intervenido la selección natural durante su adaptación a un estilo de vida domesticado seguido de una intensa selección artificial durante la formación de razas modernas (Deane, Chu, Slavney, Boyko, \& Sams, 2018). Los perros de raza pura han brindado información sobre la morfología, el comportamiento y las enfermedades, siendo la diversificación de las mismas lo que ha llevado al desarrollo de trastornos. (Sutter \& Ostrande, 2004).

Análisis realizados en 2017 indican las diferencias metabólicas entre los tamaños corporales de distintos caninos. Estas diferencias reflejan no solo la variabilidad inducida morfológicamente sino también las diferencias genéticas metabólicas específicas asociadas con la creación de razas debido a la selección artificial (Middleton et al,. 2017). Las enfermedades metabólicas son aquellas patologías causadas por anormalidades en sistemas enzimáticos implicados en el metabolismo. Las anormalidades congénitas son producidas por alteraciones genéticas que van a dar lugar a enzimas defectuosas (errores congénitos del metabolismo), mientras que las adquiridas son debidas a enfermedades de órganos endócrinos o al fallo de órganos metabólicamente actives (Mayor \& Cascales, 2006). Actualmente algunas de las enfermedades metabólicas que afectan a los caninos son la obesidad, insuficiencia renal crónica, diabetes mellitus, hipotiroidismo, desórdenes en la glándula paratiroides, hipertiroidismo, hiperadrenocorticismo, entre otras (Aillón \& Enríquez, 2013; Catherin, Moncrieff \& Guptyll, 2010). En Guatemala no se poseen estudios publicados sobre las enfermedades metabólicas que afectan a los caninos específicamente según la raza que estos sean.

El presente estudio se realizó en elHospital Veterinario de la Facultad de Medicina Veterinaria y 
Zootecnia (HVAC) de la Universidad de San Carlos siendo éste el único hospital universitario veterinario, con atención al público en el cual se reciben pacientes con todo tipo de afecciones, entre las cuales se encuentran las de origen metabólico. El propósito del estudio fue obtener una perspectiva general de la predisposición racial de ciertos desórdenes metabólicos en caninos así como la relación clínica que estos tienen con la condición corporal, estado reproductivo y sexo. Así mismo generar una fuente de información que puede ser útil para mejorar los registros del hospital y contribuir a en el ámbito de la investigación.Es importante tomar en cuenta que en el estudio la mayor parte de los caninos pertenecían a zona 12 y Mixco, zona en la que se encuentra ubicado el hospital y que forman parte de la ciudad capital respectivamente.

\section{MÉTOdos}

El presente estudio fue realizado en el Hospital Veterinario de la Facultad de Medicina Veterinaria y Zootecnica de la Universidad de San Carlos de Guatemala ubicado en la zona 12 de la ciudad capital con coordenadas UTM al Este 763330 y Norte 1613701.
Dentro de los parámetros a tomar en consideración se incluyó que fueran caninos de raza pura, su estado reproductivo, condición corporal y sexo. Se excluyó cualquier dato que no cumpliera con los parámetros antes mencionados.

Los datos fueron tomadas de todas las fichas clínicas de los pacientes correspondientes al mes de julio 2017 - enero 2018. Las mismas debían pertenecer a pacientes con diagnóstico definitivo de enfermedad metabólica siendo éstos corroborados con exámenes complementarios de laboratorio realizados en dicho centro de estudio así como el respaldo de su ficha clínica.

Para el análisis se realizó un estudio retrospectivo evaluando 406 fichas clínicas de pacientes caninos, de las cuales 48 correspondían a pacientes con un diagnóstico definitivo de enfermedad metabólica.

El análisis de datos se utilizó estadística descriptiva en la cual se clasificaron de acuerdo a raza, sexo, estado reproductivo, condición corporal y diagnóstico final.

Las pruebas diagnósticas a utilizar según la enfermedad fueron las siguientes:

\begin{tabular}{|c|c|c|c|c|c|c|c|c|c|c|c|c|}
\hline Enfermedad & \multicolumn{12}{|c|}{ Ayuda Diagnóstica } \\
\hline & Hemograma & GPT & Urianálisis & Glucosa & Creatinina & Urea & BUN & Colesterol & T4 libre & US & Ex. Fecal & Urocultivo \\
\hline Hipotiroidismo & $x$ & $x$ & $X$ & & & & & $X$ & $X$ & & & \\
\hline $\begin{array}{l}\text { Enfermedad } \\
\text { Renal Crónica }\end{array}$ & $x$ & $x$ & $x$ & & $x$ & $x$ & $x$ & & & $x$ & & $x$ \\
\hline $\begin{array}{l}\text { Diabetes } \\
\text { Mellitus }\end{array}$ & $x$ & $x$ & $x$ & $x$ & $x$ & & $x$ & $x$ & & & & \\
\hline Urolitiasis & $X$ & & $X$ & & $X$ & $X$ & $X$ & & & $X$ & & \\
\hline
\end{tabular}

III. Resultados

Tabla 1: Numero de Pacientes con Enfermedad Metabólica Según Raza

\begin{tabular}{|c|c|c|}
\hline Raza & No. & $\%$ \\
\hline Alaska Malamute & 1 & $2 \%$ \\
\hline Bichón Habanero & 1 & $2 \%$ \\
\hline Bull terrier inglés & 1 & $2 \%$ \\
\hline Collie & 1 & $2 \%$ \\
\hline Dálmata & 1 & $2 \%$ \\
\hline Dobermann & 1 & $2 \%$ \\
\hline Pastor alemán & 1 & $2 \%$ \\
\hline Rottweiler & 1 & $2 \%$ \\
\hline Samoyedo & 1 & $2 \%$ \\
\hline Bulldog Inglés & 2 & $4 \%$ \\
\hline Dachshund & 2 & $4 \%$ \\
\hline Shihtzu & 2 & $4 \%$ \\
\hline CockerSpaniel Inglés & 3 & $6 \%$ \\
\hline Golden retriever & 3 & $6 \%$ \\
\hline SRD & 4 & $8 \%$ \\
\hline Schnauzer & 4 & $8 \%$ \\
\hline French Poodle (Caniche) & 5 & $10 \%$ \\
\hline Husky siberiano & 6 & $13 \%$ \\
\hline Labrador retriever & 8 & $17 \%$ \\
\hline & 48 & $100 \%$ \\
\hline
\end{tabular}


Tabla 2: Desórdenes Metabólicos en el Hvac

\begin{tabular}{|c|c|c|}
\hline Enfermedad & No. & $\%$ \\
\hline Obesidad & 13 & $27 \%$ \\
\hline Hipotiroidismo & 13 & $27 \%$ \\
\hline Enfermedad Renal Crónica & 12 & $25 \%$ \\
\hline Urolitiasis & 8 & $17 \%$ \\
\hline Diabetes & 2 & $4 \%$ \\
\hline & 48 & $100 \%$ \\
\hline
\end{tabular}

Tabla 3: Porcentaje de Caninos con Diagnóstico de Enfermedad Metabólica Según Sexo

\begin{tabular}{|c|c|c|c|c|c|}
\hline Sexo & Pacientes & Esterilizados & No esterilizados & FI & Porcentaje \\
\hline Hembras & 27 & 11 & 13 & 1 & $56.25 \%$ \\
\hline Machos & 21 & 7 & 12 & 3 & $43.75 \%$ \\
\hline Total & 48 & 18 & 25 & 5 & $100 \%$ \\
\hline
\end{tabular}

Tabla 4: Enfermedades Metabólicas Según Raza

\begin{tabular}{|l|l|}
\hline \multicolumn{2}{|c|}{ Enfermedad según raza } \\
\hline Labrador Retriever & Obesidad \\
\hline & Hipotiroidismo \\
\hline & Diabetes Mellitus \\
\hline & Urolitiasis \\
\hline & Enfermedad Renal Crónica \\
\hline Husky Siberiano & Obesidad \\
\hline & Hipotiroidismo \\
\hline & Urolitiasis \\
\hline & Enfermedad Renal Crónica \\
\hline & Enfermedad Renal Crónica \\
\hline Frencha Poodle & Obesidad \\
\hline & Hipotiroidismo \\
\hline & Diabetes Mellitus \\
\hline & Urolitiasis \\
\hline Enermedad Renal Crónica \\
\hline Schnauzer & Obesidad \\
\hline & Hipotiroidismo \\
\hline
\end{tabular}

Tabla 5: Cantidad y Porcentaje de Caninos Según Condición Corporal

\begin{tabular}{|c|c|c|}
\hline Condición Corporal & Cantidad & $\%$ \\
\hline 1 & 2 & $4 \%$ \\
\hline 2 & 2 & $4 \%$ \\
\hline 3 & 4 & $8 \%$ \\
\hline 4 & 11 & $23 \%$ \\
\hline 5 & 5 & $10 \%$ \\
\hline 6 & 2 & $4 \%$ \\
\hline 7 & 5 & $10 \%$ \\
\hline 8 & 14 & $29 \%$ \\
\hline 9 & 2 & $4 \%$ \\
\hline $\mathrm{FI}$ & 1 & $2 \%$ \\
\hline & 48 & $100 \%$ \\
\hline
\end{tabular}


Tabla 6: Cantidad de Pacientes con Enfermedad Metabólica y Su Localidad

\begin{tabular}{|l|c|}
\hline \multicolumn{1}{|c|}{ Localidad } & Pacientes \\
\hline Zona 12 & 7 \\
\hline Zona 13 & 4 \\
\hline Zona 7 & 4 \\
\hline Zona 8 & 1 \\
\hline Zona 10 & 1 \\
\hline Zona 11 & 4 \\
\hline Zona 17 & 2 \\
\hline Zona 18 & 1 \\
\hline Zona 19 & 2 \\
\hline Zona 21 & 1 \\
\hline Zona 5 & 1 \\
\hline Mixco & 7 \\
\hline Villa Nueva & 2 \\
\hline Villa Hermosa & 1 \\
\hline Villa Canales & 1 \\
\hline San José Pinula & 1 \\
\hline Carretera a Fraijanes & 2 \\
\hline Carretera San Lucas Sacatepéquez & 1 \\
\hline Chinautla & 2 \\
\hline Escuintla & 1 \\
\hline Jalapa & 1 \\
\hline Sacatepéquez Total & 1 \\
\hline & 48 \\
\hline
\end{tabular}

\section{Discusión}

El grupo de estudio se conformó por 406 pacientes del área de Medicina Interna del HVAC, de los cuales 48 tuvieron un diagnóstico final de enfermedad metabólica. Entre las dificultades para la obtención del diagnóstico final estuvo la situación económica por parte de los propietarios, la cual no permitió en algunos casos la realización de ayudas diagnósticas complementarias así como el seguimiento clínico del paciente.

De acuerdo a Gought y Thomas (2004)la mayoría de las enfermedades genéticas reconocidas del perro se heredan de forma autosómica recesiva. Esto puede ser debido a la endogamia, pero también se debe a la dificultad de identificar y eliminar los rasgos recesivos en los programas de mejoramiento. Es común observar que perros cruzados son más sanos que los de raza pura,relacionándose con la endogamia, la cual tiende a enmascarar el efecto de muchos genes recesivos.

La fijación del fenotipo y el apareamiento de individuos relacionados han dado lugar a patrones de enfermedad específicos de raza y variaciones en la esperanza de vida de éstos. (Fleischer, Sharkey, Maeley, Ostrander \& Martínez, 2008). Por esto mismo la presencia de enfermedades metabólicas en ciertas razas puras cada vez es más caracterizable y presente en Guatemala.

Dentro del estudio se determinó la condición corporal de los distintos caninos atendidos en el HVAC ya que la obesidad además de ser considerada una enfermedad metabólica se relaciona con el desarrollo de otras afecciones de este tipo. En los pacientes se pudo determinar que solamente el $33 \%$ mostraba una condición corporal adecuada en rangos entre $4-5$ de 9 y un $47 \%$ presentó una condición corporal en rangos entre 6 -9 de 9, siendo éstos últimos ya considerados con sobrepeso y con mayor exposición a un síndrome metabólico. La obesidad canina se asocia con el desarrollo de resistencia a la insulina, perfiles lipídicos alterados e hipertensión leve, que se alivian con la pérdida de peso. Además, los perros con sobrepeso son más propensos a padecer diabetes mellitus, mientras que la sobrealimentación de por vida conduce a un sobrepeso, trastornos metabólicos y una disminución de la esperanza de vida. (Gought \& Thomas, 2004) (Mori et al., 2010). Estudios realizados en Estados Unidos indican que el sobrepeso y la obesidad afectan aproximadamente a 1 de cada 5 perros, habiendo aumentado su prevalencia a un 37\% en perros desde 2007. En perros, el 62\% con sobrepeso presentan hipotiroidismo (García, 2012) y 5.18 veces más tendientes a padecer insuficiencia renal (Aillón \& Enríquez, 2013).

Aunque el número de caninos esterilizados resultó ser menor que el de no esterilizados es importante mencionar que los estrógenos regulan la saciedad a nivel del sistema nervioso central, de modo que tras la castración, sobre todo en las hembras, se produce un aumento del apetito y reducción del índice metabólico, siendo los animales castrados los de mayor probabilidad de padecer sobrepeso u obesidad y por ende una enfermedad metabólica (González \& Serrano, 2017). 
Dentro del estudio se observó que las hembras tuvieron un porcentaje superior que los machos a padecer una enfermedad metabólica correlacionándose con estudios de medicina humana, en los cuales las endocrinopatías se presentan con mayor frecuencia en mujeres (González, 2015). Por ejemplo, entre las endocrinopatías con mayor predisposición en las hembras se encuentra el hipotiroidismo. (Malaga Vet Summit, 2017).

Según los resultados el hipotiroidismo y la obesidad resultaron ser losdesórdenes de mayor frecuencia en el HVAC con un $27 \%$ cada una.El hipotiroidismo es una enfermedad con un rango de aparecimiento medio, usualmente afectando entre los 2-6 años (Gought \& Thomas, 2004). Entre las razas con mayor predisposición a la enfermedad y de mayor presencia presentadas en el HVAC se encontraron elLabrador Retriever, Husky Siberiano, Schnauzer y French Poodle.

El protocolo diagnóstico de Ettinger para el hipotiroidismo (Catherin, Moncrieff \& Guptyll, 2010) difiere con el HVAC solamente en que no se miden electrolitos ni TSH, debido a que no se encuentra como parte del protocolo la medición de los mismos y no existe dicha prueba en el país. Así mismose realizael urianálisis con el fin de no incluir como enfermedad principal la enfermedad de Cushing, la cual presenta signos similares al hipotiroidismo pero densidades urinarias bajas en el $85 \%$ de los pacientes (DiBartola, 2010).

De acuerdo a Mori et al. (2010) los Schnauzer miniatura son la primera raza canina reportada en Estados Unidos que suele padecer de hiperlipidemia primaria presentando niveles elevados de triglicéridos plasmáticos y colesterol total. Ambos, signos de hiperlipidemia la cual puede ser provocada por el hipotiroidismo. En el HVAC se presentó un $8 \%$ de caninos raza Schnauzer entre los cuales existía diagnóstico de hipotiroidismo. Dentro de las pruebas a realizarse en dicho hospital son los niveles de colesterol, más no de triglicéridos por lo cual el diagnóstico de hiperlipidemia puede llegar a ser inconcluso.Por esta razón sería recomendable agregar como protocolo la medición de estos en todos los animales con énfasis en los Schnauzer. Según un estudio de la red de hospitales estadounidenses Banfield Pet Hospital, el hipotiroidismo se da en 1 de cada 200 perros, además, el 61\% de los perros hipotiroideos padecen también sobrepeso u obesidad. (Aillón \& Enríquez, 2013).

Seguido del hipotiroidismo, la enfermedad renal crónica resultó ser la segunda enfermedad de mayor aparecimiento con un 25\%. Las nefropatías crónicas son las enfermedades renales más frecuentes en la clínica de los pequeños animales y una de las principales causas de mortalidad en pacientes de edad avanzada. Ocurre en perros de todas las edades, sexos y razas (Suárez, 2007). De acuerdo con los resultados del HVAC tanto el Husky Siberiano, Labrador Retriever y French Poodle fueron razas que presentaron dicha afección. Según estudios, en esta enfermedad existe una prevalencia general que oscila entre el 0.5 y el $7 \%$ en los perros. (Suárez, 2007).De acuerdo al protocolo deEttinger (DiBartola, 2010) el HVAC difiere en la medición de la presión sanguínea, la cual no se realiza en el mismo debido a la falta de equipo específico. Esta es aconsejable realizarla ya que la hipertensión suele ser una secuela del fallo renal. Estudios demuestran que la incidencia de hipertensión en perros con fallo renal suele ser del 30 - 93\% siendo aquellos con afección glomerular los que en mayor riesgo se encuentran (DiBartola, 2010).

Luego la urolitiasisresultó ser la tercera enfermedad de mayor prevalencia en el HVAC la cual puede estar asociada a distintos factores tales como raza, sexo, edad y dieta. SegúnRobles (Robles, 2016)dentro de las razas de mayor tendencia a padecer esta afección se encuentran el Labrador Retriever, Schnauzery Poodle, mismas presentadas en el estudio con mayor presentación en el HVAC y algunas de ellas con diagnóstico final de urolitiasis. De acuerdo a Robles (2016) estudios anteriores indican que dicha enfermedad tiende a afectar a las razas más pequeñas con mayor frecuencia que las grandes pudiendo relacionarse esto con su menor volumen de orina, y el menor número de micciones, por lo tanto mayor concentración de minerales. La predisposición racial a tipos específicos de minerales sugiere una base genética. (Robles, 2016). Las principales pruebas diagnósticas realizadas en el HVAC cuando se sospecha de urolitiasis son ultrasonido abdominal, urianálisis para determinar qué tipo de cristales se encuentran, urocultivo y análisis del urolito Seguido de éstas se realizan algunas pruebas básicas como medición de creatinina sanguínea y perfiles proteicos para descartar cualquier tipo de falla renal asociada al urolito. Muchas veces el análisis del urolito es realizado sólo si el dueño puede pagarlo y algunas veces se limita a las pruebas mencionadas anteriormente. El análisis del mismo es para saber su composición ya que dependiendo de ella puede detectarse otro tipo de afecciones tales como lasalteraciones hepáticas e hipercalciuria.De mismo modo a partir del análisis de éste se puede saber qué manejo dietético debe seguirse y así evitar la recurrencia de la enfermedad.

En cuanto a la diabetes mellitus se encuentra ampliamente distribuida a nivel de razas de perros tendientes a padecerla. Según los pacientes del HVAC tanto la raza Labrador Retriever como French Poodle resultaron con un diagnóstico concluyente de DM. De acuerdo a Fall et al. (2007)las diferencias significativas de raza, sexo y edad indican que la variación genética podría hacer que las razas sean más o menos susceptibles a diferentes tipos de DM. Se requiere de 
mayor investigación, principalmente sobre las diferencias entre las distintas características, y los estudios específicos de raza. Así mismo ese mismo estudio realizado en Estados Unidos concluyó que los samoyedos (y todas aquellas razas nórdicas como el Husky Siberiano), los Schnauzers miniatura y el caniche miniatura tenían un alto riesgo de padecer DM; razas presentes dentro de éste mismo estudio. También dentro de ésta enfermedad algunas hembras de raza específica presentan mayor riesgo de sufrirla, sin embargo razas como el Labrador Retriever no poseen preferencia de sexo en cuanto a padecer dicha afección. (Fall, Hansson, Hedhammar, Kampe \& Egenvall, 2007)

Dentro del diagnóstico de diabetes mellitus realizado en el HVAC se encuentra la presencia de signos clínicos clásicos como la poliuria, polidipsia, polifagia y pérdida de peso. Una vez encontrados estos se procede a la medición de glucosa tanto en orina como en sangre. Así mismo se mide la densidad urinaria, hemograma, GPT, perfil renal, creatinina y colesterol en sangre. La batería de pruebas se hace con el fin de descartar cualquier tipo de afección que pueda estar causándola o incrementando su presencia. De acuerdo a Ettinger (Nelson, 2010) otras pruebas a realizar son medición de lipasa pancreática, insulina, concentración de progesterona sérica en hembras sin esterilizar y urocultivo. Sin embargo éstas últimas generalmente no se realizan en el HVAC ya que con las pruebas básicas es posible determinar en gran medida la enfermedad así como por razones económicas antes mencionadas.

\section{Abreviaturas}

HVAC: Hospital Veterinario de la Facultad de Medicina Veterinaria y Zootecnia

GPT: Transaminasa glutámica pirúvica

BUN: Nitrógeno ureico en sangre

US: Ultrasonido

SRD: Sin raza definida

Fi: Final inconcluso

\section{BiBLIOGRAFÍA}

1. Aillón, A., \& Enríquez, S., (2013). Determinación de la prevalencia de las enfermedades metabólicas (cardíacas, hepáticas, renales y obesidad) en caninos del distrito metropolitano de Quito en el período enero - junio del año 2012. 1(1). 14 -31. Recuperado de: http://dspace.udla.edu.ec/handle/ 33000/2893

2. Deane, P., Chu, E., Slavney, A., Boyko, A., \& Sams, A., (2018). Direct-to-consumer DNA testing of 6,000 dogsreveals 98.6-kb duplication associated with blue eyes and heterochromia in Siberian Huskies. Plos Genetics. 1(1): 2 Recuperado de: https://www. researchgate.net/publication/328083829_Direct-to-
consumer_DNA_testing_of_6000_dogs_reveals_986 $\mathrm{kb}$ duplication_associated_with_blue_eyes_and_he terochromia_in_Siberian_Huskies

3. Ettinger, S., \& Feldman, E., (2010) Clinical approach and Laboratory Evaluation. DiBartola, S., Ettinger Textbook of Veterinary Internal. (pp 1716-1730) Elsevier Saunders.

4. Ettinger, S., \& Feldman, E., (2010). Diabetes mellitus. Nelson, R., Ettinger Textbook of Veterinary Internal. (pp.1590). Elsevier Saunders.

5. Ettinger, S., \& Feldman, E., (2010) Hipotiroidismo. Catherin, J., Moncrieff, S., Guptyll, L., Ettinger Textbook of Veterinary Internal. (pp 1540) Elsevier Saunders.

6. Fall, T., Hansson, H., Hedhammar, A., Kampe, O., \& Egenvall, A., (2007). Diabetes Mellitus in a Population of 180,000 Insured Dogs; Incidence, Survival, and Breed Distribution. (1) (27) 1209- 1216. Recuperado de: https://onlinelibrary.wiley.com/doi/ epdf/10.1111/j.1939-1676.2007.tb01940.x

7. Fleischer, S., Sharkey, M., Maeley, K., Ostrander, E., \& Martínez, M., (2008). Pharmacogenetic and Metabolic Differences Between Dog Breeds: Theirlmpacton Canine Medicine and the Use of the Dog as a Preclinical Animal Model. Springer Link. 10 (1): 110-119. Recuperado de: https://link.springer. com/article/10.1208/s12248-008-9011-1

8. García, C., (2012). Estudio sobre el incremento de enfermedades crónicas en perros y gatos. Banfield Pet Hospital, Recuperado de: http://www.stateof pethealth.com/pdf/State_of_Pet_Health_2012.pdf

9. González, F., \& Serrano, $\bar{C}$., (2017). Incidencia de enfermedades endocrinas en caninos entre los años 2013-2016 en un hospital veterinario universitario de Chile. Revista chilena endocrinología. 10 (3): 90-94. Recuperado de: http://revistasoched.cl/3_2017/3.pdf

10. González, J., (2015). Relación de las patologías caninas más frecuentes que se presentan en la clínica de pequeños animales en la zona noroeste de la Comunidad de Madrid, con las variables edad, raza, sexo y tamaño. Universidad Complutense de Madrid. 1(1). 47. Recuperado de: https://eprints.ucm.es/33266/1/T36414.pdf

11. Gough, A., \& Thomas, A., (2004). Breed Predispositions to Disease in Dogs and Cats. United Kingdom, England. Blackwell Publishing LTD.

12. Málaga Vet Summit. Esterilización: del mito a la realidad.(2017) FYCMA. 1(1) 6-7. Recuperado de: http://www.colvet.es/sites/default/files/2018-02/ Dossier_Vet_Summit_2017_Conclusiones_v02.pdf

13. Mayor, F., \& Cascales, M., (2006). Enfermedades Metabólicas. 1 (1). 11. Recuperado de: http://200. 72.129.100/hso/guiasclinicasneo/17_Enfermedades _Metabolicas_Congenitas.pdf. 
14. Middleton, R., Lacroix, S., Scott-Boyer, M., Dordevic, N., Kennedy, A., Slusky, A., et al. (2017). Metabolic Differences between Dogs of Different Body Sizes. PMC. 1(1): 10 Recuper ado de: https://www.ncbi.nlm.nih.gov/pmc/articles/PMC568 4564/\#sec5title

15. Mori, N., Leea, P., Muranakaa, S., Sagaraa, F., Takemitsua, H., Nishiyamaal, et al. (2010). Predisposition for primary hyperlipidemia in Miniature Schnauzers and Shetland sheepdogs as compared to other canine breeds. Elsevier. 88(3) 394 - 399. Recuperdo de: https://www.Science direct.com/science/article/abs/pii/S0034528809002 951

16. Suárez, M., (2007) Manejo de la enfermedad renal crónica. RECVET. 2 (01-04). 1. Recuperado de: http://www.veterinaria.org/revistas/recvet/n01a0407/ 01a040706.pdf

17. Sutter, N., \& Ostrande, E., (2004). Dog star rising: the canine genetic system. Nature Reviews. 1(1): 1 Recuperado de: https://nature.com/articles/nrg1492

18. Robles, B., (2016). Situación actual de la urolitiasis canina en cuatro hospitales de la ciudad de Guatemala. FMVZ. USAC. 1(1) pp. 17. Recuperado de: http://www.repositorio.usac.edu.gt/5266/1/Tesis \%20Med\%20Vet\%20Blanca\%20E\%20Robles\%20Pa redes.pdf 